\title{
The $\boldsymbol{t}-\boldsymbol{J}$ Model in a Strong Magnetic Field
}

\author{
A. SHERMAN* \\ Institute of Physics, University of Tartu, Riia 142, 51014 Tartu, Estonia
}

\begin{abstract}
The normal-state energy spectrum of the two-dimensional $t-J$ model in a homogeneous perpendicular magnetic field $B$ is investigated using the Mori projection operator technique. The density of states at the Fermi level as a function of $1 / B$ reveals both high- and low-frequency oscillations. The high-frequency oscillations correspond to large Fermi surfaces, while the low-frequency components are related to van Hove singularities in the Landau subbands, which stem from their bending due to strong electron correlations. Frequencies of the low-frequency components are of the same order of magnitude as those observed in underdoped cuprates. These components become dominant if smoothing processes are involved. It is shown that despite increased distances between subbands the pseudogap affects only slightly the frequency of density of states oscillations.
\end{abstract}

DOI: 10.12693/APhysPolA.127.213

PACS: 71.10.Fd, 71.27.+a, 71.70.Di, 74.72.Gh

\section{Introduction}

The decreased quantum oscillation frequencies, which are observed in the mixed state of underdoped cuprates [1], are usually interpreted with no regard for strong electron correlations (see, e.g., $[2,3]$ ). However, it is known that these crystals are characterized by strong electron correlations. In this work we consider the twodimensional (2D) $t-J$ model of $\mathrm{Cu}-\mathrm{O}$ planes in the homogeneous magnetic field $B$, which is perpendicular to the model plane. The used approach allows us to take proper account of strong electron correlations and to consider large enough clusters in moderate magnetic fields. We found that in the range of hole concentrations $0.08<x<0.18$ the DOS at the Fermi level oscillates with changing $B$. The oscillations have components with frequencies differing by an order of magnitude. The high-frequency components are connected with large Fermi surfaces, while the low-frequency components are related to van Hove singularities in the Landau subbands, which traverse the Fermi level with changing $B$. These van Hove singularities are linked with bending the Landau subbands due to strong correlations. Frequencies of slow components are of the same order of magnitude as quantum oscillation frequencies in underdoped cuprates. Hence, the $t-J$ model supplemented with a mechanism, which smears out high-frequency oscillations, is able to interpret observed low frequencies of quantum oscillations. To estimate the effect of the pseudogap we considered a weakly correlated system with gaps at the antinodal points in the spectrum. Despite the gaps increase distances between the Landau subbands, they change only slightly quantum oscillation frequencies.

\section{Model}

The Hamiltonian of the 2D $t-J$ model in the perpendicular magnetic field reads

\footnotetext{
*e-mail: alexei@fi.tartu.ee
}

$$
\begin{aligned}
H & =\sum_{\boldsymbol{l l ^ { \prime } \sigma}} t_{\boldsymbol{l} \boldsymbol{l}^{\prime}} \exp \left(\mathrm{i} \frac{e}{\hbar} \int_{\boldsymbol{l}}^{\boldsymbol{l}^{\prime}} \boldsymbol{A}(\boldsymbol{r}) \mathrm{d} \boldsymbol{r}\right) a_{\boldsymbol{l}_{\sigma}}^{\dagger} a_{\boldsymbol{l}^{\prime} \sigma} \\
& +\frac{1}{2} \sum_{\boldsymbol{l \boldsymbol { l } ^ { \prime }}} J_{\boldsymbol{l} \boldsymbol{l}^{\prime}}\left(s_{\boldsymbol{l}}^{z} s_{\boldsymbol{l}^{\prime}}^{z}+s_{\boldsymbol{l}}^{+} s_{\boldsymbol{l}^{\prime}}^{-}\right),
\end{aligned}
$$

where $2 \mathrm{D}$ vectors $\boldsymbol{l}$ and $\boldsymbol{l}^{\prime}$ label sites of a square lattice, $\sigma= \pm 1$ is the projection of the hole spin, $a_{l_{\sigma}}^{\dagger}=|\boldsymbol{l} 0\rangle\langle\boldsymbol{l} \sigma|$ and $a_{\boldsymbol{l} \sigma}=|\boldsymbol{l} \sigma\rangle\langle\boldsymbol{l} 0|$ are hole creation and annihilation operators with the empty $|\boldsymbol{l} 0\rangle$ and singly occupied $|\boldsymbol{l} \sigma\rangle$ site states. The hole kinetic energy $H_{k}$ contains the hopping matrix element $t_{l l^{\prime}}$ and the Peierls phase factor [4] with the vector potential $\boldsymbol{A}(\boldsymbol{r})$. The exchange term includes the exchange constant $J_{l l^{\prime}}$ and the spin- $\frac{1}{2}$ operators $s_{\boldsymbol{l}}^{z}=\frac{1}{2} \sum_{\sigma} \sigma|\boldsymbol{l} \sigma\rangle\langle\boldsymbol{l} \sigma|$ and $s_{\boldsymbol{l}}^{ \pm}=|\boldsymbol{l}, \pm 1\rangle\langle\boldsymbol{l}, \mp 1|$ of localized spins. The Zeeman term is omitted, since for the considered fields and exchange constants in cuprates it is two orders of magnitude smaller than the exchange term.

In the following consideration we suppose that only nearest neighbor hopping $t$ and exchange $J$ constants are nonzero. In the Landau gauge $\boldsymbol{A}(\boldsymbol{l})=-B l_{y} \boldsymbol{x}$, where $l_{y}$ is the $y$ component of the site vector $\boldsymbol{l}$ and $\boldsymbol{x}$ is the unit vector along the $x$ axis. Hence the exponential in the kinetic term of the Hamiltonian can be written as $\mathrm{e}^{\mathrm{i} \boldsymbol{\kappa}_{a} \boldsymbol{l}}$, $\boldsymbol{\kappa}_{\boldsymbol{a}}=-\frac{e}{\hbar} B a_{x} \boldsymbol{y}, \boldsymbol{a}$ is the vector connecting neighboring sites. We shall restrict our consideration to the fields satisfying the condition $\frac{e}{\hbar} B a^{2}=2 \pi \frac{n^{\prime}}{n}, a=|\boldsymbol{a}|, n$ and $n^{\prime}<n$ are integers with no common factor. In this case the kinetic term of the Hamiltonian defines its translation properties $-H_{k}$ is invariant with respect to translations by the lattice period along the $x$ axis and by $n$ lattice periods along the $y$ axis. To retain this symmetry we apply the periodic Born-von Karman boundary conditions to the sample with $N_{x}$ sites along the $x$ axis and $n N_{y}$ sites along the $y$ axis. The boundary conditions define the set of allowed wave vectors, and the momenta $\boldsymbol{\kappa}_{\boldsymbol{a}}$ coincide with some of them. This fact simplifies the Fourier transformation over $\boldsymbol{l}$ in $H_{k}$,

$$
H_{k}=t \sum_{\boldsymbol{K} \boldsymbol{a} \sigma} \mathrm{e}^{\mathrm{i} \boldsymbol{K} a} a_{\boldsymbol{K}-\boldsymbol{\kappa}_{a}, \sigma}^{\dagger} a_{\boldsymbol{K} \sigma} .
$$

It is convenient to split the Brillouin zone into $n$ stripes of the width $\frac{2 \pi}{n a}$, which are oriented parallel to the $x$ axis. 
If we select the lowest stripe with $-\frac{\pi}{a}<K_{y} \leq-\frac{\pi}{a}+\frac{2 \pi}{n a}$, and denote wave vectors in it as $k$, momenta in the entire Brillouin zone can be described as $\boldsymbol{k}+j \boldsymbol{\kappa}$. Here $0 \leq j \leq n-1$ and $\boldsymbol{\kappa}=\frac{2 \pi}{n a} \boldsymbol{y}$. In these notations the kinetic energy acquires the form $H_{k}=\sum_{\boldsymbol{k} \sigma} \boldsymbol{A}_{\boldsymbol{k} \sigma}^{\dagger} \boldsymbol{h}_{\boldsymbol{k}} \boldsymbol{A}_{\boldsymbol{k} \sigma}$, where the summation over $\boldsymbol{k}$ is performed over the selected stripe, $\boldsymbol{A}_{\boldsymbol{k} \sigma}^{\dagger}=\left(a_{\boldsymbol{k} \sigma}^{\dagger}, a_{\boldsymbol{k}+\boldsymbol{\kappa}, \sigma}^{\dagger}, \ldots a_{\boldsymbol{k}+(n-1) \boldsymbol{\kappa}, \sigma}^{\dagger}\right)$ and the matrix $\boldsymbol{h}_{\boldsymbol{k}}$ has the following elements:

$$
h_{\boldsymbol{k} j j^{\prime}}= \begin{cases}2 \cos \left(k_{y} a+\frac{2 j \pi}{n}\right), & j=j^{\prime}, \\ \mathrm{e}^{-\mathrm{i} k_{x} a}, & j=j^{\prime}-n^{\prime}, \\ \mathrm{e}^{\mathrm{i} k_{x} a}, & j=j^{\prime}+n^{\prime}, \\ 0, & \text { in other cases. }\end{cases}
$$

In this equation the matrix indices $j$ and $j^{\prime}$ are determined modulo $n$.

The Hermitian matrix (3) can be diagonalized by the unitary transformation $\boldsymbol{U}_{\boldsymbol{k}}$. Since the kinetic energy defines symmetry properties of the total Hamiltonian (1), the operators $\alpha_{\boldsymbol{k} m \sigma}=\sum_{j=0}^{n-1} U_{\boldsymbol{k} j m}^{*} a_{\boldsymbol{k}+j \boldsymbol{\kappa}, \sigma}$, $0 \leq m \leq n-1$, form a basis for a representation of the symmetry group of the Hamiltonian, and, therefore, they are an appropriate starting point for calculations. We shall calculate Green's function $G(\boldsymbol{k} m \bar{t})=$ $-\mathrm{i} \theta(\bar{t})\left\langle\left\{\alpha_{\boldsymbol{k} m \sigma}^{\dagger}(\bar{t}), \alpha_{\boldsymbol{k} m \sigma}\right\}\right\rangle$, where the averaging over the grand canonical ensemble and the operator time dependence are determined by the Hamiltonian $\mathcal{H}=H-$ $\mu \sum_{\boldsymbol{l}}|\boldsymbol{l} 0\rangle\langle\boldsymbol{l} 0|$ with the chemical potential $\mu$. Since $\alpha_{\boldsymbol{k} m \sigma}$ is constructed from Hubbard operators, in these calculations we use the Mori projection operator technique [5]. In this approach, the Fourier transform of $G(\boldsymbol{k} m \bar{t})$ is represented by the continued fraction

$$
G(\boldsymbol{k} m \omega)=\frac{\left\langle\left\{\alpha_{\boldsymbol{k} m \sigma}, \alpha_{\boldsymbol{k} m \sigma}^{\dagger}\right\}\right\rangle}{\omega-E_{0}-\frac{V_{0}}{\omega-E_{1}-\frac{V_{1}}{\ddots}}} .
$$

For every $\boldsymbol{k}$ and $m$ we calculated terms $E_{0}, V_{0}$ and $E_{1}$, which approximate (4) by two poles. The multitude of lower poles for different $\boldsymbol{k}$ and $m$ form Landau subbands originated from the spin-polaron band. In the underdoped case these subbands cross the Fermi level, and, therefore, they are of primary interest for us. Equations for $E_{0}, V_{0}$ and $E_{1}$ are given in [6].

\section{Results}

The hole DOS, $\rho(\omega)=-\frac{1}{N \pi} \sum_{\boldsymbol{k} m} \operatorname{Im} G(\boldsymbol{k} m \omega)$, as a function of frequency and magnetic field reveals oscillations near the Fermi level for $B \neq 0$. An example of these oscillations is shown in Fig. 1. Here and hereafter we set $t$ and $a$ as units of energy and length, respectively. In the calculations we set $J / t=0.2$ and the temperature $T=0$. The oscillations are observed in the concentration range $0.08<x<0.18$. Outside of this range the Fermi level falls on strong maxima of the DOS, where the oscillations are lost against the background.

In the used two-pole approximation, in the considered range of $x$ the zero-field Fermi surfaces are large. The main oscillation frequency of $\rho(0)$ as a function of

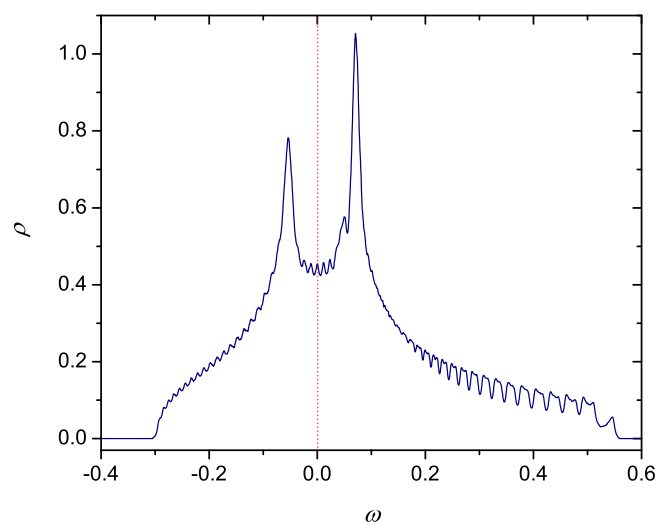

Fig. 1. The hole DOS in the case $n=63, n^{\prime}=1$ and $x=0.11$. The Fermi level is shown by the red dashed line.

$\frac{1}{B}=\frac{e a^{2}}{2 \pi \hbar} \frac{n}{n^{\prime}}$ conforms with such Fermi surfaces - for the case $x=0.14$ the frequency $F \approx 10 \mathrm{kT}$ for $a=4 \AA$ (an approximate distance between copper sites in $\mathrm{Cu}-\mathrm{O}$ planes, see Fig. 2a). However, along with these high-frequency oscillations a modulation with a period which is larger by an order of magnitude is also observed. To reveal the respective low-frequency oscillations the data were smoothed with the FFT filter method, which allows one to suppress high-frequency components. The result is shown in Fig. 2b. The frequency of these oscillations is of the order of $1 \mathrm{kT}$, which is comparable to the dominant frequency of quantum oscillations in underdoped $\mathrm{YBa}_{2} \mathrm{Cu}_{3} \mathrm{O}_{6+x}$ [1]. Thus, supplemented with a mechanism, which smears out high-frequency oscillations, the $t-J$ model is able to explain the low-frequency quantum oscillations observed in cuprate perovskites.

The reason for the appearance of the low-frequency modulation in Fig. 2a is the following. Let us plot the Landau subband $m$ in the stripe $-\pi+\frac{2 \pi}{n} m<K_{y} \leq$ $-\pi+\frac{2 \pi}{n}(m+1),-\pi<K_{x} \leq \pi$ of the entire Brillouin zone. As a result the hole dispersion looks like stairs with steps ascending from smaller to larger energies when $K_{y}$ changes from $-\pi$ to $\pi$. In the uncorrelated case energies in a step experience only weak oscillations when $k_{x}$ varies from $-\pi$ to $\pi$ in the Landau gauge. The traverse of the Fermi level through such subbands leads to oscillations in the DOS with a nearly constant amplitude. Strong correlations lead to a bend of the subbands along the $x$ direction. This bending, which is seen in Fig. 3, leads to the appearance of van Hove singularities. They supplement the DOS oscillations with the amplitude modulation, since the singularities vary with a period, which is larger than the distance between steps in the stairs in Fig. 3 (notice that strong correlations modify the stairs with changing the Fermi level). Thus, the low-frequency modulation and oscillations in Fig. 2 are connected with traversing the Fermi level through the sequence of van Hove singularities in the Landau subbands. We suppose that the inclusion of a next-nearest-neighbor hopping does not qualitatively change these results. 


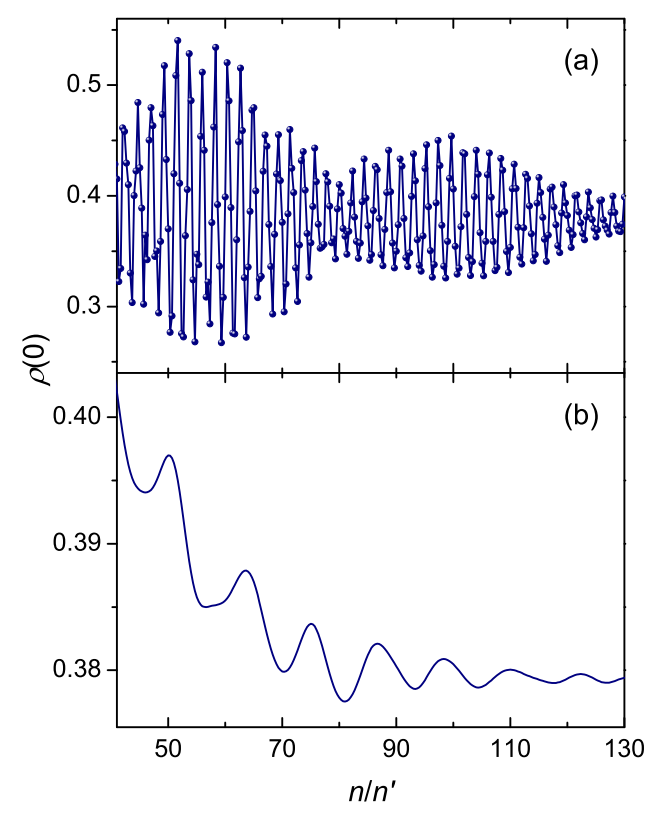

Fig. 2. (a) The DOS at the Fermi level as a function of $\frac{n}{n^{\prime}}=\frac{2 \pi \hbar}{e a^{2}} \frac{1}{B}$ for $n^{\prime}=3$ and $x=0.14$. Calculated values are shown by symbols, connecting lines are a guide to the eye. (b) The same data smoothed by the FFT filter method.

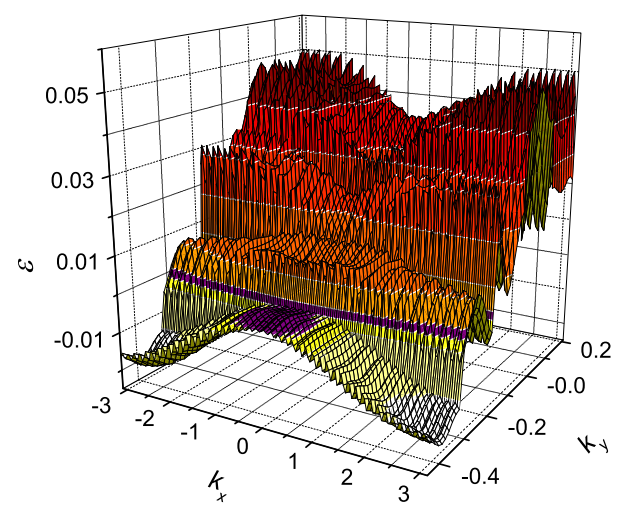

Fig. 3. The dispersion of hole states near the Fermi level for $n=47, n^{\prime}=1$ and $x=0.14$. The Fermi level is shown by the purple (dark) contour. Only subbands near the Fermi level are shown.

The used two-pole approximation does not describe the pseudogap and Fermi arcs, which can influence the quantum oscillation frequency. To clarify their effect we have compared $\rho(0)$ in a weakly correlated system with and without gaps in the energy spectrum near antinodal points. The dispersion with the gaps is described in the same manner as in [7]. The calculations are reduced to the diagonalization of a Hamiltonian of the type of (2) with large amount of hopping integrals, which are necessary for the description of the gaps. The result is shown in Fig. 4. The gaps increase the energy interval between Landau subbands, as seen from the enlarged oscillation amplitude. However, the frequency of oscillations in $\rho(0)$ changes only slightly.

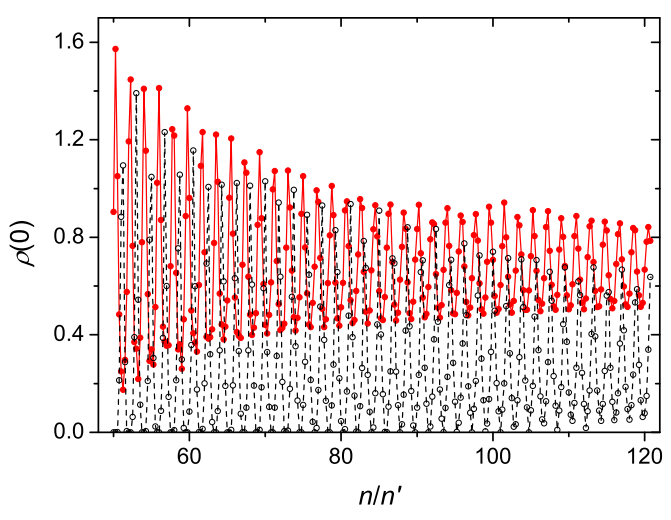

Fig. 4. The DOS at the Fermi level in the absence of gaps at the antinodal region (red solid circles and line) and with gaps of the magnitude $0.2 t$ and Fermi arcs of the length $1 / a$ (black open circles and dashed line). $n^{\prime}=4$.

\section{Summary}

In summary, we have considered the $2 \mathrm{D} t-J$ model of $\mathrm{Cu}-\mathrm{O}$ planes of cuprate perovskites in the perpendicular homogeneous magnetic field. In the range of hole concentrations $0.08<x<0.18$ the DOS at the Fermi level $\rho(0)$ shows oscillations as a function of the inverse field $1 / B$. The oscillations have high- and low-frequency components. The latter components are connected with the bending of the Landau subbands near the Fermi level, which is a result of strong electron correlations. The bending leads to the appearance of van Hove singularities in the subbands, which produce the low-frequency components in $\rho(0)$ with changing $B$. Frequencies of these components $F \approx 1 \mathrm{kT}$ are of the same order of magnitude as dominant quantum oscillation frequencies observed in underdoped cuprate perovskites. Thus, being supplemented with a mechanism smearing out the high-frequency components, the model is able to account for these experiments. We argue that the pseudogap influence only slightly the quantum oscillation frequency.

\section{Acknowledgments}

This work was supported by the European Regional Development Fund (project TK114) and by the Estonian Scientific Foundation (grant ETF9371).

\section{References}

[1] S.E. Sebastian, N. Harrison, G.G. Lonzarich, Rep. Prog. Phys. 75, 102501 (2012).

[2] A.J. Millis, M. Norman, Phys. Rev. B 76, 220503(R) (2007).

[3] A. Melikyan, O. Vafek, Phys. Rev. B 78, 020502(R) (2008).

[4] R. Peierls, Z. Phys. 80, 763 (1933).

[5] H. Mori, Prog. Theor. Phys. 34, 399 (1965).

[6] A. Sherman, M. Schreiber, Phys. Lett. A 377, 2979 (2013).

[7] A. Sherman, M. Schreiber, J. Supercond. Nov. Magn. 25, 1833 (2012). 